\title{
QUALITY IMPROVEMENT Sleep is the best medicine: How rest facilities and EnergyPods can improve staff wellbeing
}

\author{
Authors: Eoin Dore, ${ }^{\mathrm{A}}$ Daniel Guerero, ${ }^{\mathrm{B}}$ Thomas Wallbridge, ${ }^{\mathrm{C}}$ Angela Holden, ${ }^{\mathrm{D}}$ Mehreen Anwar, ${ }^{\mathrm{E}}$ Alison Eastaugh, ${ }^{\mathrm{F}}$ \\ David Desai ${ }^{A}$ and Sarbjit Clare ${ }^{G}$
}

\section{Introduction}

Healthcare workers' wellbeing is critical to the NHS. Night shifts have a big impact on their physical and mental health. Recently there has been national funding for rest facilities.

Methods

An EnergyPod was installed in the acute medical unit. The study comprised two surveys: one quantitative survey performed prior to the pod introduction and repeated 3 months after; and one qualitative survey performed immediately after pod use.

\section{Results}

We observed more staff taking breaks of 30 minutes after the pod introduction ( $37 \%$ before vs $69 \%$ after). Of users, $81 \%$ felt more alert and $83 \%$ were more energised. Half of the respondents felt more able to drive after use.

The feedback showed three themes: appreciation of designated space away from clinical areas, relaxation and improved interdisciplinary cohesion.

Conclusion

We have shown consistent data that rest is important for wellbeing. We recommend the use of EnergyPods in high acuity areas.

KEYWORDS: wellbeing, sleep, rest facilities, EnergyPod, multidisciplinary cohesion

DOI: 10.7861/fhj.2020-0261

\section{Introduction}

Junior doctor wellbeing is important to the function of the NHS. Frequently working long hours with little support acts as

Authors: ${ }^{A}$ anaesthetic core trainee and wellbeing chair, Sandwell and West Birmingham NHS Trust, Birmingham, UK; ${ }^{\text {B }}$ foundation year-2 doctor, Sandwell and West Birmingham NHS Trust, Birmingham, UK; ${ }^{C}$ anaesthetic core trainee, Sandwell and West Birmingham NHS Trust, Birmingham, UK; ' core medical trainee, Sandwell and West Birmingham NHS Trust, Birmingham, UK;

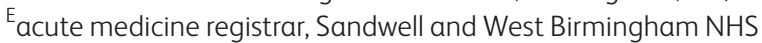
Trust, Birmingham, UK; Felderly medicine registrar, Sandwell and West Birmingham NHS Trust, Birmingham, UK; ${ }^{G}$ acute medicine consultant, Sandwell and West Birmingham NHS Trust, Birmingham, UK a significant driver of healthcare worker anxiety and fatigue. ${ }^{1}$ Night shifts are known to be physically and mentally taxing as it requires one to function accurately and efficiently at a time when the human body is programmed for sleep. ${ }^{2}$ The physiological challenge of long night shifts can be detrimental to both healthcare worker wellbeing and patient care. Night shifts have been linked to a significant increase in cardiovascular risk, obesity, diabetes and primary sleep disorders in healthcare workers. ${ }^{3}$ It increases their chances of mistakes on the job, with reports associating long 12 -hour day or night shifts with twice as many accidents as an 8 -hour shift. ${ }^{4}$ Nightshift work in healthcare professionals was associated with road traffic accidents after the shift due to fatigue while driving home. Sleep deprivation also impairs recent learning as consolidation occurs during rapid eye movement (REM) sleep and, therefore, night shift work also has implications on training. ${ }^{5,6}$ The psychomotor effects of night shifts can also negatively impact patient care through its manifestation from poor decision making, reduced procedural accuracy, inadvertent medicines administration, error in drug dosing calculations and greater risk-taking behaviours.

There has been a recent shift in perspective with the significance of healthcare worker wellbeing finally being recognised by several large bodies. This has been helped by publications such as the British Medical Association's (BMA's) Fatigue and facilities charter as well as General Medical Association's Caring for doctors caring for patients which both advocate for staff wellbeing practices. 7,8 A consistent component of these documents is a drive to change the stigma and cultural view of sleeping or resting at work. ${ }^{9}$ This has recently been supported by the secretary of state for health through the allocation $£ 10$ million for the provision of rest facilities as outlined in the BMA's Fatigue and facilities charter. ${ }^{10}$ Napping and breaks have now been recognised by the Royal College of Physicians (RCP), Royal College of Nursing and BMA as crucial for patient safety. ${ }^{11}$ The RCP recommends taking multiple naps to counteract fatigue on night shifts. ${ }^{12}$ A systematic review showed that 15-20 minute restorative naps can have significant benefits in terms of level of alertness and cognitive performance. ${ }^{13}$ This view was subsequently reinforced by a further two systematic reviews in 2018 and 2020. .4,15 $^{-1}$

The management team at the Sandwell and West Birmingham NHS Trust took a proactive approach to wellbeing and sought out strategies to actively promote it. A recent survey of local junior doctors ( $n=47$ ) showed only $29 \%$ had access to rest areas or a staff room, primarily highlighting a lack of facilities in high acuity areas such as acute medicine. Most respondents only took 15-30 minutes of break compared with the recommended 
60 minute allocation. Recurring reasons cited included a lack of facilities as well as judgement from other clinical staff. There was a pre-existing doctors' mess in a separate building, however, due lack of proximity and subsequent safety concerns, only one out of 59 people surveyed reported that they had used it.

To address this issue, one strategy was the decision to trial the use of Energy Pods in high acuity areas. An EnergyPod, costing around $€ 13,000$ and measuring approximately $2.2 \mathrm{~m}$ by $1.4 \mathrm{~m}$, is a specially designed chair which has been purpose built for napping in the workplace. It is ergonomically designed and equipped with a privacy visor as well as built in speakers and timed waking function to optimise the napping experience and function of staff. ${ }^{16}$

\section{Aims}

This study aimed to assess the impact of the provision of an EnergyPod and sleep education on the wellbeing of medical and nursing staff in high acuity areas.

\section{Methods}

The study took place over a 4-month period from July to October 2019 and was set in the acute medical unit of a 304 bed district general hospital, City Hospital in Birmingham. The acute medical unit consists of 48 beds and is typically staffed by 10 nurses, five healthcare assistants and three junior doctors at night. The project was led by the deputy medical director (who is also an acute physician) and, together with enthusiastic junior doctors, created a project group to deliver and analyse the results on this initiative.

The EnergyPod was installed in a designated room on the acute medical unit and its appropriate use was widely publicised to the staff of this department (Fig 1). This was communicated locally through announcements at handover meetings and board rounds, as well as trust wide via multiple magazine articles and videos on the intranet. Sleep hygiene education was also incorporated into regular junior doctor teaching. This widespread dissemination of information encouraged consultants to actively promote the use of the pod.

Sleep education focused on highlighting the hazards of sleep deprivation as well as a summary of the sleep advice detailed by Dr Michael Farquhar, consultant paediatrician and sleep specialist. ${ }^{11}$ It also focused on reversing the negative connotations associated with napping on the job and emphasised the importance of taking breaks.

The study design employed the use of serial cross-sectional surveys with a focus on qualitative data collection with some quantitative components. The study comprised two surveys: one
Table 1. Pre- and post-EnergyPod results

\begin{tabular}{|c|c|c|c|}
\hline Question & Answer & $\begin{array}{l}\text { Before pod } \\
\text { introduction, } \\
n=93, n(\%)\end{array}$ & $\begin{array}{l}\text { After pod } \\
\text { use, } n=68 \\
n(\%)\end{array}$ \\
\hline \multirow[t]{2}{*}{ What is your job role? } & Doctor & $35(38)$ & $33(49)$ \\
\hline & Nurse & $58(62)$ & $35(51)$ \\
\hline $\begin{array}{l}\text { Are you able to take } \\
\text { your allocated break } \\
\text { time in } 12 \text {-hour shifts? }\end{array}$ & Yes & $34(37)$ & $47(69)$ \\
\hline \multirow[t]{2}{*}{$\begin{array}{l}\text { How alert do you feel } \\
\text { after your night shift? }\end{array}$} & $\begin{array}{l}\text { Alert / } \\
\text { very alert }\end{array}$ & $23(25)$ & $45(66)$ \\
\hline & Not alert & $70(75)$ & $23(34)$ \\
\hline
\end{tabular}

quantitative survey that was performed prior to the introduction of the EnergyPod and subsequently repeated 3 months after; and one qualitative survey that was performed immediately after pod use.

The quantitative study focused on assessing prevailing levels of fatigue and alertness on long shifts in the pre- and post-pod phase. The qualitative survey collected data on the length of use as well as immediate impact on alertness and energy levels. However, the primary focus of this survey was to collect descriptive data on individual experience.

\section{Results}

A total of 93 participants responded to the pre-pod survey and 68 responded to the post-pod introduction survey. After the introduction of the pods, there was a significant improvement in taking breaks and alertness at the end of the shift (Table 1). Users also reported an improvement in perceived safety of driving after night shifts (Table 2). Responses also suggested that the sleep pod was a suitable rest environment, with a positive impact on wellbeing, which they would recommend to a colleague (Table 2).

Prior to the EnergyPod, greater than $80 \%$ of workers reported that stress and fatigue had a negative impact on their personal life. Following 3 months' use of the pod, $74 \%$ of users reported some positive impact on their physical or psychological health and their ability to manage stress. Eighty-seven per cent of respondents thought the EnergyPod would help with morale and wellbeing.

A total of 31 responses were received immediately post-use of the EnergyPod. There were three major themes developing from the descriptions received.
Fig 1. The EnergyPod installed in the dedicated room.

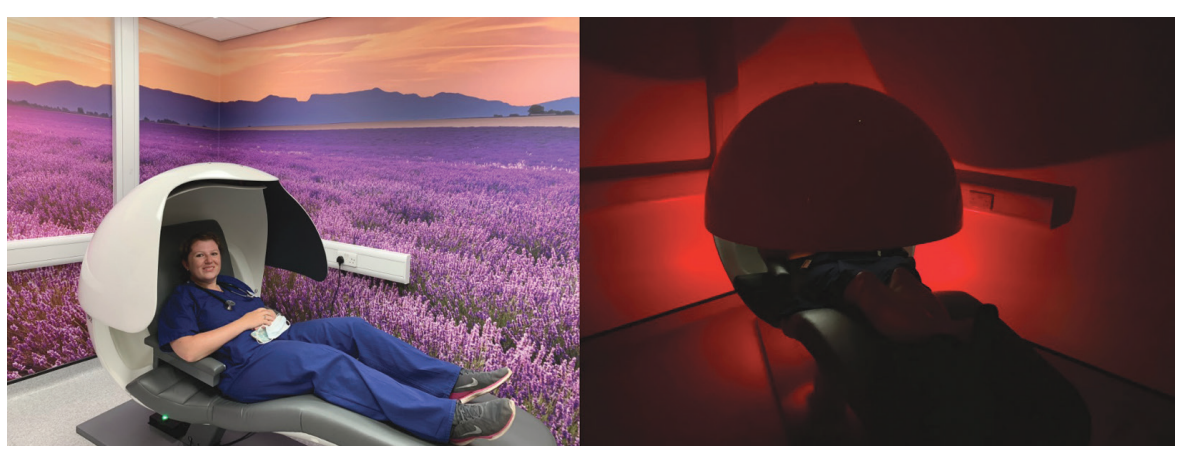


Table 2. Post-EnergyPod introduction results

\begin{tabular}{|c|c|c|}
\hline Question & Answer & $\begin{array}{l}\text { After pod use, } \\
n=36, n(\%)\end{array}$ \\
\hline \multirow{2}{*}{$\begin{array}{l}\text { Do you feel safer to drive after } \\
\text { using the EnergyPod? }\end{array}$} & Yes & 19 (53) \\
\hline & $\begin{array}{l}\text { No / don't } \\
\text { know }\end{array}$ & $17(47)$ \\
\hline \multirow{2}{*}{$\begin{array}{l}\text { Do you think the availability } \\
\text { of the EnergyPod will help in } \\
\text { wellbeing }\end{array}$} & Yes & 32 (89) \\
\hline & $\begin{array}{l}\text { No / don't } \\
\text { know }\end{array}$ & $4(11)$ \\
\hline \multirow{2}{*}{$\begin{array}{l}\text { Did you find the EnergyPod to } \\
\text { be a suitable rest environment? }\end{array}$} & Yes & $24(68)$ \\
\hline & $\begin{array}{l}\text { No / don't } \\
\text { know }\end{array}$ & $12(32)$ \\
\hline \multirow{2}{*}{$\begin{array}{l}\text { Would you recommend the } \\
\text { EnergyPod to a colleague }\end{array}$} & Yes & $34(94)$ \\
\hline & $\begin{array}{l}\text { No / don't } \\
\text { know }\end{array}$ & $2(6)$ \\
\hline
\end{tabular}

The first theme communicated was a general appreciation of a designated, quiet, non-clinical area for breaks. This subsequently encouraged staff to take breaks, with senior staff opting to designate defined break times to juniors. Supporting quotes included '[It's] really nice having something that is not a doctor's office. [It] feels like I can actually switch off for 20 minutes,' and '[It] helped me after stress to get away from the ward.'

The second major theme was an appreciation of the relaxing effect of the EnergyPod with improved energy and stress reduction. Supporting quotes included ' [I] feel calmer like I'm able to process thoughts better,' '[From] my time in the pod, I felt relaxed and ready to do my job all over again. Amazing,' and 'Very calming and relaxing during mid-shift to ease stress.' Eighty-four per cent of respondents felt more energised and $81 \%$ felt more alert.

The third theme was improved interdisciplinary cohesion due to positive interactions of encouraging each other to rest. There was increased recognition on stressors and fatigue of different multidisciplinary team members and subsequent encouragement to take rest. Participants described an overall sense of increased comradery between teams while on shift. Multiple respondents said it was 'Nice to see something for nurses.' Ninety-four per cent of respondents would recommend the EnergyPod to a colleague.

\section{Discussion}

The EnergyPod was beneficial to staff wellbeing. Crucially, they are not designed for deep sleep: they enable a short period "power nap' due to a pre-set timed function. Users are unlikely to drop into deeper sleep stages in this period, preventing a 'sleep hangover.'11 This power nap has been shown to improve alertness and energy, as we also observed in our data. ${ }^{13}$ Further useful features include sensory distraction with music, ambient lighting and vibration, which enables accelerated relaxation. This is significant given the time pressures in high acuity areas where rest time is minimal.

The use of both qualitative and quantitative data allowed the analysis of both cohort and individual use. The quantitative data showed the need for pods and the great majority (88\%) were in favour of keeping the facilities, with $94 \%$ recommending to a colleague.
The feedback from the EnergyPod showed three major themes: space away from the clinical workspace, relaxation and interdisciplinary cohesion. Users benefited from a dedicated space for relaxation, easily accessible within the unit. We also observed that around half of our respondents felt more able to drive after a shift during which they had used the pod. McClelland et al presented a worrying trend among trainees, with $84 \%$ of trainees feeling too tired to drive after a night shift. ${ }^{17}$ The hope is that by promoting and providing rest facilities, trainees will be able to reduce their personal risk of road traffic accidents.

The third theme was not one we expected; however, the unintended consequence of improved team cohesion is a positive outcome. We found respondents from all the multidisciplinary team (MDT) reporting an improvement in their wellbeing and a positive impact on their physical as well as psychological health. Initially, the project had been primarily focused on junior doctors, but this project naturally spilt over into the MDT, bringing members of the team closer together.

What is difficult to convey in data are the cultural behaviours of a department. Firstly, the physical presence of an EnergyPod sent a very strong message to the workers on the floor that management teams and leaders care about the staff and their wellbeing, and that was incredibly powerful. Secondly, the promotion of 'it is okay to take a rest' from senior clinicians who actively promoted, if not mandated, this sent a clear message to all juniors that this was accepted and expected behaviour, promoting safety for everyone. Typically, many clinicians do not take breaks, with the main reasons often cited as workload pressures or that the act exhibits weakness. Top down strong leadership was a key enabler in the success of this project and cultural shift of the mindset of taking breaks.

Although our data show significant positive impact since the introduction of the EnergyPod, the contribution of culture change and education on proper sleep hygiene should not be underestimated. Given the lack of rest spaces beforehand, a significant confounder to the evaluation of the impact of the EnergyPod itself was the establishment of a designated rest space. It is also not possible to distinguish between the effects of the pod and sleep hygiene advice. The project focused on subjective staff wellbeing but did not analyse the effect of rest on patient safety (for example, by looking at prescribing errors during a night shift). This could be a further expansion of the project.

There are a few criticisms of the EnergyPod, mainly associated with its cost, cleanliness and capacity. The EnergyPods are priced at $£ 13,000$ with alternatives such as a bed or reclining chair costing significantly less. However, none of these alternatives incorporate its salient features; for instance, a recliner chair or bed would not provide the immersive yet controlled rest environment provided by the EnergyPod. Through multiple uses, the pod can become unhygienic and therefore relies on users to sanitise after use. The EnergyPod is also much larger than alternatives, requiring its own room, potentially limiting the number of pods that can be incorporated into the unit.

\section{Conclusion}

The introduction of an EnergyPod with sleep education was shown to have a positive impact on medical and nursing staff. Most users reported increased energy and alertness after use, with many appreciating the quiet and relaxing space away from the clinical area. It facilitated increased team cohesion as the culture 
shifted to encourage dedicated breaks with coverage of clinical responsibilities. The staff in the acute medical unit have benefited immensely from the rest facilities and EnergyPod implementation. We recommend night shift staff take a mandatory 20-minute rest to improve their alertness and accuracy.

\section{Supplementary material}

Additional supplementary material may be found in the online version of this article at www.rcpjournals.org/fhj:

S1 - Questionnaire.

\section{Acknowledgements}

We would like to thank Dr Rose Bailey for use of the photograph.

\section{Funding}

Funding for the energy pod was provided by Sandwell and West Birmingham NHS Trust.

\section{References}

1 Kecklund G, Axelsson J. Health consequences of shift work and insufficient sleep. BMJ 2016;355:i5210.

2 Fallis WM, McMillan DE, Edwards MP. Napping during night shift: practices, preferences, and perceptions of critical care and emergency department nurses. Crit Care Nurse 2011;31:e1-11.

3 British Medical Association. Fatigue and sleep deprivation - the impact of different working patterns on doctors. London: BMA, 2018.

4 Salminen S. Shift work and extended working hours as risk factors for occupational injury. The Ergonomics Open Journal 2010;3:14-8.

5 Stickgold R. Sleep-dependent memory consolidation. Nature 2005;437:1272-8.

6 Stickgold R, James L, Hobson JA. Visual discrimination learning requires sleep after training. Nat Neurosci 2000;3:1237-8.
7 British Medical Association. Fatigue and facilities charter. London: BMA, 2018.

8 West M, Coya D. Caring for doctors: caring for patients. General Medical Council, 2019.

9 Rimmer A. NHS needs culture shift regarding sleeping at work. BMJ 2016;354:i3982.

10 British Medical Association. Fatigue and facilities FAQs. BMA, 2018. www.bma.org.uk/media/1077/bma_fatiguea_andf-facilities_ investment_faqs_oct_2019.pdf

11 Farquhar M. Fifteen-minute consultation: problems in the healthy paediatrician-managing the effects of shift work on your health. Arch Dis Child Educ Pract Ed 2017;102:127-32.

12 Horrocks N, Pounder R, RCP Working Group. Working the night shift: preparation, survival and recovery-a guide for junior doctors. Clin Med 2006:6:61-7.

13 Ruggiero JS, Redeker NS. Effects of napping on sleepiness and sleep-related performance deficits in night-shift workers: a systematic review. Biol Res Nurs 2014;16:134-42.

14 Martin-Gill C, Barger LK, Moore CG et al. Effects of napping during shift work on sleepiness and performance in emergency medical services personnel and similar shift workers: a systematic review and meta-analysis. Prehosp Emerg Care 2018;22(suppl 1):47-57.

15 Dutheil F, Bessonnat B, Pereira B et al. Napping and cognitive performance during night shifts: a systematic review and metaanalysis. Sleep 2020;43:zsaa109.

16 MetroNaps. The EnergyPod. MetroNaps, 2020. www.metronaps. com/\#energypod

17 McClelland L, Holland J, Lomas JP, Redfern N, Plunkett E. A national survey of the effects of fatigue on trainees in anaesthesia in the UK. Anaesthesia 2017;72:1069-77.

Address for correspondence: Dr Eoin Dore, Acute Medicine, Birmingham City Hospital, Dudley Road, Birmingham B18 7QH, UK.

Email: eoin.dore@nhs.net

Twitter: @DoreEoin 